group involving the receptive component of the Receptive-Expressive Emergent Language Scale (REEL), all subsections of the Preschool Language Scale (PLS), the Revised Peabody Picture Vocabulary Test (PPVT-R), and the verbal comprehension/language-processing section of the Stanford-Binet. These deficits were recognized by 3 years of age, but required specific quantitative testing by a psychologist or speech and language pathologist for their detection. (Baker RC et al. Neurodevelopmental outcome of infants with viral meningitis in the first three months of life. Clin Pediatr June 1996;35:295-301). (Reprints: Raymond C Baker MD, OSB 4, Children's Hospital Medical Center, 3333 Burnet Avenue, Cincinnati, Ohio 45229).

COMMENT. Children with enteroviral meningitis in early infancy should be monitored carefully for impairments of language development, particularly receptive language. Those with recognized deficits should receive increased language stimulation in the home prior to enrollment in school.

\title{
IN UTERO COCAINE EXPOSURE AND INFANT BEHAVIOR
}

The effects on neurobehavior in 20 infants with prenatal exposure to cocaine, alcohol, marijuana, and cigarettes, compared to 17 infants exposed to alcohol and/or marijuana and cigarettes without cocaine and 20 drug-free infants, were assessed using the Neonatal Intensive Care Unit Network Neurobehavioral Scale at Brown University School of Medicine, Women and Infants Hospital, Providence, RI. Cocaine-exposed infants showed increased rone and motor activity, more jerky movements, startles, tremors, back arching, and signs of central nervous system and visual stress than unexposed infants. Visual and auditory following responses, and birth weight and length of cocaine-exposed infants were also reduced. (Napiorkowski B, Lester BM et al. Effects of in utero substance exposure on infant neurobehavior. Pediatrics July 1996;98:71-75). (Reprints: Barry M Lester PhD, Women and Infant's Hospital, 101 Dudley St, Providence, RI 02905).

COMMENT. Meconium testing was used to confirm lack of illicit drug use in the unexposed group. Positive meconium or urine assays were found in 5 women who had denied prenatal drug use. Urine toxicology can detect cocaine within 1 to 4 days of last use. Cocaine-exposed infants had neurobehavioral changes especially involving increased tone and motor activity. Synergistic effects of cocaine with alcohol and marijuana could not be ruled out.

Dose-related effects of cocaine on 3-week neurobehavior were demonstrated in a study at Children's Hospital, Boston, MA. Comparing 38 heavily exposed infants, 73 lightly exposed, and 94 unexposed, after controlling for covariates, a significant dose effect was observed, heavily exposed infants showing poorer regulation of arousal and greater excitability at 3-week examination but not in the first few days of life. (Tronick EZ et al. Late dose-response effects of prenatal cocaine exposure on newborn neurobehavioral performance. Pediatrics July 1996;98:76-83). (Reprints: Edward Z Tronick PhD, Children's Hospital, 300 Longwood Ave, Boston, MA 02115).

Since regulation of arousal and attention are important to learning, infants exposed to cocaine in utero may be expected to show decreased developmental scores and to have attention deficit disorders in childhood. (Ped Neur Briefs Feb 1996;10:9-10). 